\title{
Dynamics of the higher-order structure of chromatin
}

\author{
Ping Chen, Guohong $\mathrm{Li}^{\bowtie}$ \\ Institute of Biophysics, Chinese Academy of Sciences, Beijing 100101, China \\ $\bowtie$ Correspondence: liguohong@sun5.ibp.ac.cn
}

\begin{abstract}
Eukaryotic DNA is hierarchically packaged into chromatin to fit inside the nucleus. Dynamics of the chromatin structure plays a critical role in transcriptional regulation and other biological processes that involve DNA, such as DNA replication and DNA repair. Many factors, including histone variants, histone modification, DNA methylation and the binding of non-histone architectural proteins regulate the structure of chromatin. Although the structure of nucleosomes, the fundamental repeating unit of chromatin, is clear, there is still much discussion on the higher-order levels of chromatin structure. Identifying the structural details and dynamics of higher-order chromatin fibers is therefore very important for understanding the organization and regulation of gene activities. Here, we review studies on the dynamics of chromatin higherorder structure and its relationship with gene transcription.
\end{abstract}

KEYWORDS chromatin, higher-order structure, dynamics, transcriptional regulation

The accessibility of eukaryotic DNA is dependent on the packing density of chromatin fibers in the nucleus. It has been clear that the dynamics of higher-order chromatin compaction is one of the key regulators of gene transcription. Crystal Xray studies have defined the structure of nucleosomes, the basic units of chromatin, at high resolution; they consist of $147 \mathrm{bp}$ of DNA wrapping around an octamer of histones (two copies each of $\mathrm{H} 2 \mathrm{~A}, \mathrm{H} 2 \mathrm{~B}, \mathrm{H} 3$ and $\mathrm{H} 4$ ) approximately 1.7 times (Luger et al., 1997). The primary structure of chromatin, a "beads-on-a-string" nucleosomal fiber with nucleosomes connected by linker DNA, is highly compacted into a condensed $30 \mathrm{~nm}$ chromatin fiber which is typically regarded as the secondary structure (Robinson and Rhodes, 2006). Further compaction of chromatin fibers may form other higher-level chromatin structures, but so far details of these structural levels remain obscure. Although it is clear that the hierarchical formation of chromatin higher-order structure includes intra-nucleosome interactions, nucleosome-nucleosome interactions, intra/inter-chromatin fiber interactions and interactions between chromatin and chromatin architectural proteins, the interplay between these interactions and the detailed arrangement of nucleosomes within chromatin higher-order structures, including $30 \mathrm{~nm}$ fibers, still remains largely uncharacterized. In this review, we focus on recent progresses in elucidating the detailed structure of higherorder chromatins and their structural dynamics in eukaryotic gene regulation.

\section{STRUCTURE OF THE $30 \mathrm{~nm}$ CHROMATIN FIBERS: SOLENOID VS ZIG-ZAG MODEL}

Early examinations of chromatin higher-order structure in nuclei and chromosomes in situ have yielded disappointingly litte information. It is very difficult to adequately resolve nucleosomes and linker DNA in compact chromatin under conditions in the nucleus, and to trace the paths of chains of nucleosomes. Many investigators have focused on native chromatins isolated from nuclei. Based on electron microscopy (EM) studies of native chromatin fibers, two models have been proposed for $30 \mathrm{~nm}$ fibers; the one-start solenoid model and the two-start Zig-Zag model (Fig. 1). The solenoid model suggests that the nucleosome array folds into a onestart helix with adjacent nucleosomes connected by bent linker DNA (Widom and Klug, 1985). In the second model, nucleosmes are arranged in a Zig-Zag mode to form two stacks of nucleosomes connected by straight linker DNA which criss-crosses between each stack (Woodcock et al., 1984; Williams et al., 1986). Although the chromatin isolated 

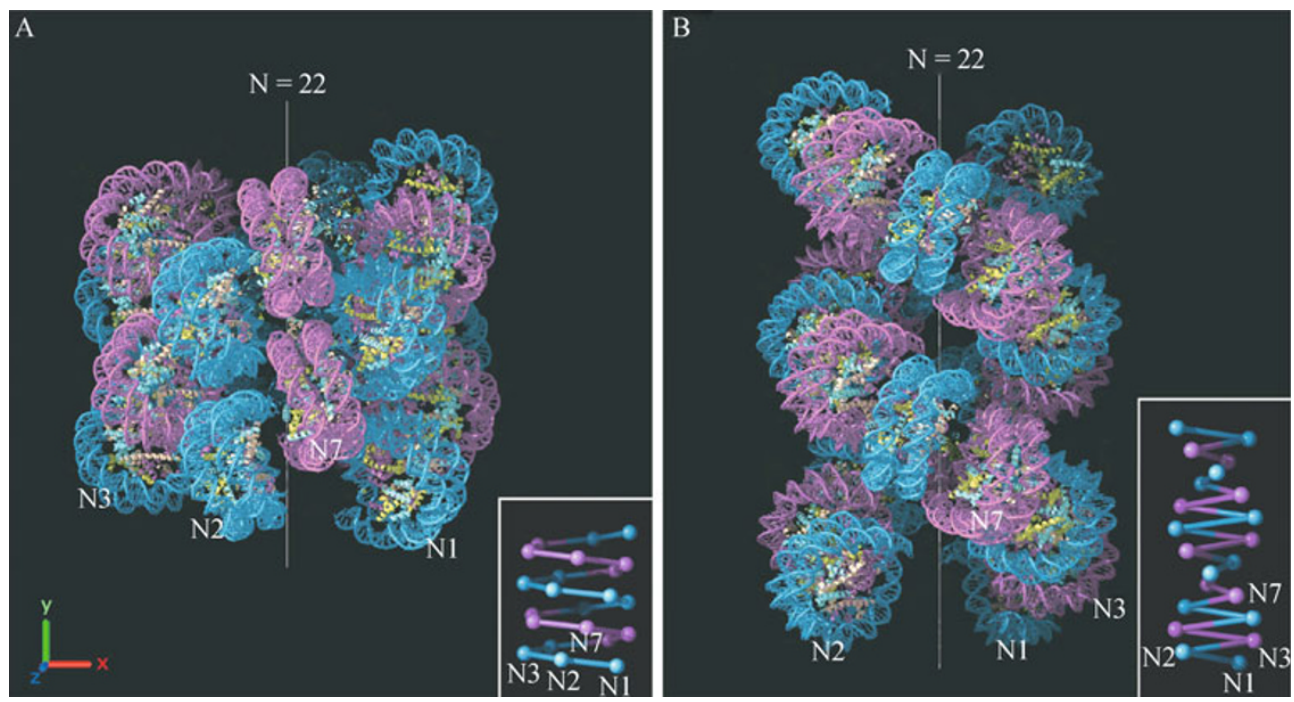

Figure 1. Solenoid (A) VS Zig-zag (B) model for $\mathbf{3 0} \mathbf{~ n m ~ c h r o m a t i n ~ f i b e r s . ~ T h e ~ p o s i t i o n s ~ o f ~ t h e ~ f i r s t , ~ s e c o n d , ~ t h i r d , ~ a n d ~ s e v e n t h ~}$ nucleosomes (N1, N2, N3, and N7) in the linear DNA sequence are marked in the insets to clarify the arrangement of nucleosomes in the chromatin fiber in both models. (from Robinson et al. (2006), Copyright (2006) National Academy of Sciences, U.S.A.).

from nuclei is mostly native, it is very heterogeneous with different DNA sequences/linker lengths and different histone compositions/modifications. The irregular spacing between nucleosomes in native chromatin makes it difficult to define the detailed structure of chromatin.

To distinguish between these two models, an in vitro system using strong "nuclesome positioning" sequences (Dorigo et al., 2004) has recently been developed to generate well-defined and regular spacing nuclesomal arrays. Using this system, Richmond and colleagues have successfully reconstituted tetranucleosomal arrays on a "widom 601 nucleosome positioning" sequence, and propose a two-start crossed-linker model based on crystal X-ray diffraction (Schalch et al., 2005). The two-row Zig-Zag ribbon conformation was also observed on much longer reconstituted arrays by EM studies and cross-linking assays (Dorigo et al., 2004). However, the crystal structure was determined for a tetranucleosomal array, which is too short to form a solenoid structure. A very short nucleosome repeat length (NRL, 167 $\mathrm{bp}$ ), which is uncommon in nature, was used. Furthermore, the data were obtained at a very high concentration of $\mathrm{Mg}^{2+}$ $(120 \mathrm{mM})$ in the absence of linker histone. Rhodes and colleagues have proposed an alternative one-start interdigitated solenoid structure based on EM/cryo-EM studies (Robinson et al., 2006). They analyzed chromatin fibers reconstituted under more native conditions in the presence of linker histone with a wide range of NRLs (177-237 bp) and in low divalent salt concentrations $\left(1.6 \mathrm{mM} \mathrm{Mg}^{2+}\right)$. It has been reported that $\mathrm{Mg}^{2+}$ plays an important role in chromatin condensation in vivo, with the average concentration in nuclei changing from $3 \mathrm{mM}$ at interphase to $17 \mathrm{mM}$ at metaphase (Strick et al., 2001). Although some studies have proposed that the linker histone is dispensable for the compaction of reconstituted nucleosomal arrays under high concentrations of $\mathrm{Mg}^{2+}$ (Schalch et al., 2005; Shogren-Knaak et al., 2006), Rhodes and colleagues have found that both the linker histone and NRL play a critical role in the formation of chromatin higher-order structures (Routh et al., 2008). The linker histone has also been considered to be essential for $30 \mathrm{~nm}$ chromatin fiber formation based on early EM studies on native chromatin (Thoma et al., 1979). The precise structural role of linker histone in the $30 \mathrm{~nm}$ chromatin fiber still remains to be determined. It is also worth noting the physiologic relevance of the $30 \mathrm{~nm}$ chromatin fiber. Although there is a lack of in vivo evidence for the existence of $30 \mathrm{~nm}$ fibers, fibers assembled under physiologic conditions in vitro can be used to define the mechanism of chromatin dynamics. Elucidating the structure of unmodified $30 \mathrm{~nm}$ fibers is essential for understanding the structural principles of chromatin dynamics and their relationship with gene regulation.

\section{CHROMATIN DYNAMICS AND GENE REGULATION}

During DNA replication and gene transcription, the accessibility of DNA is believed to be highly regulated by the dynamic nature of chromatin higher-order structures. DNA methylation, histone modifications, histone variants and the binding of non-histone architechtural proteins have been shown to be crucial in regulating the dynamics of chromatin structures. Acetylation of core histones prevents chromatin from folding into $30 \mathrm{~nm}$ fibers and reduces the ability of chromatin to selfassemble into higher-order structures. Peterson and colleagues have reported that acetylation of histone $\mathrm{H} 4$ on lysine 16 
(H4K16), a mark of transcription activation, can inhibit higherorder chromatin folding induced by $\mathrm{Mg}^{2+}$ (Shogren-Knaak etal., 2006). The acetylation of H3K56 results in increased breathing of DNA ends (Neumann et al., 2009; Watanabe et al., 2010). The H3K56Q mutation (to mimic acetylation) affects the oligomerization of subsaturated nucleosomal arrays, but not of saturated arrays, suggesting that H3K56 acetylation plays an important role in keeping nucleosomefree regions accessible to DNA binding proteins (Watanabe etal., 2010). The histone variant H2A.Z has been shown to increase the size of acidic patches on the surface of nucleosomes and facilitate chromatin folding into higherorder structures (Fan et al., 2004). H2A.Bbd, which is largely excluded from the female inactive $X$ chromosome in mammals, plays an important role in destabilizing nucleosomes and unfolds the chromatin fiber locally. The incorporation of H2A.Bbd into chromatin also leads to greater efficiency in the acetylation of histone tails by p300 and results in an increase in transcriptional activation (Angelov et al., 2004).

Chromatin binding proteins, including linker histones and non-histone architectural proteins, play a critical role in assembling and maintaining unique chromatin higher-order structures. Linker histones have long been proposed to be involved in the formation of higher-order chromatin structure and in gene regulation (Zlatanova et al., 2000; Catez et al., 2006). Interaction of chromatin with linker histone $\mathrm{H} 1$ limits the mobility of nucleosomes and the accessibility of chromosomal DNA. The in vitro chromatin study has demonstrated that the formation of highly compacted $30 \mathrm{~nm}$ chromatin fibers requires the incorporation of histone $\mathrm{H} 1$ and the deacetylation of core histones (Li et al., 2010). The dynamics of H1 binding to chromatin is regulated by its subtypes and post-translational modifications. Phosphorylation of histone $\mathrm{H} 1$ has been found to weaken the binding of $\mathrm{H} 1$ to nucleosomes, and provides a signal for the disassembly of chromatin higherorder structures during interphase (Hendzel et al., 2004). It is still not well known whether the different subtypes have distinct roles or if they regulate specific promoters. We have recently shown that the malignant brain tumor (MBT) protein L3MBTL1 can compact nucleosomal arrays dependent on mono- and di-methylation of H4K20 and H1bK26, and negatively regulate the expression of a subset of genes (Trojer et al., 2007). In addition to the modification activity on core histone tails, in which polycomb repressive complex 1 (PRC1) can ubiquitylate H2AK119 and PRC2 methylates $\mathrm{H} 3 \mathrm{~K} 27$, respectively, the Polycomb group (PcG) proteins can also directly compact reconstituted nucleosomal arrays in vitro to repress gene transcription (Francis et al., 2004). Heterochromatin protein 1 (HP1) has been demonstrated to bind to methylated $\mathrm{H} 3$ tails via their $\mathrm{N}$-terminal chromodomain and chromatin fiber-containing histone variant $\mathrm{H} 2 \mathrm{~A} . \mathrm{Z}$, and to compact or bridge chromatin fibers into higher-order heterochromatin structures by forming dimers via its chromoshadow domain (Bannister et al., 2001; Fan et al., 2004).
The study of structural dynamics for higher-order chromatin is critical for understanding the molecular mechanisms of epigenetic effects on gene transcription. It is very important to correlate the dynamics of chromatin strutctures with their gene functions. However, most recent studies on higher-order chromatin structures are based on nucleosomal arrays reconstituted in vitro on recombinant strong "nucleosome positioning" DNA sequences. It is difficult to integrate information on chromatin structure and biologic function using this system. We have successfully developed a new in vitro system using native DNA sequences and RSF/NAP-1 for reconstituting more natural chromatin fibers which can be used directly for both structural and functional analyses ( $\mathrm{Li}$ et al., 2010). Based on this system, we have demonstrated that transcription factors RAR/RXR can bind to the PEPCK promoter embedded in highly compacted chromatin in vitro. Following the binding of RAR/RXR, recruitment of histonemodifying enzyme p300 and ATP-dependent chromatin remodeler SWI/SNF disassemble the highly compacted chromatin fiber which then undergoes nucleosomal rearrangement to allow the activation of the PEPCK gene (Li et al., 2010).

\section{PERSPECTIVES AND CONCLUSIONS}

In spite of the large volume of information now available on the dynamics of chromatin structure, much more detailed structural information on higher-order chromatin, especially on the $30 \mathrm{~nm}$ chromatin fiber and its relationship with gene regulation, still needs to be further clarified. As mentioned above, more native experimental systems need to be developed to integrate the structure and dynamics of higher-order chromatin and gene function. In addition, improved techniques may be needed to help clarify and perhaps redefine chromosomal structures. Cryo-EM, which enables direct high-resolution observation of cell structures in a close-to-native state, can be used to analyze the detailed arrangement of nucleosomes in higher-order chromatin. Dubochet and colleagues have used cryo-EM and image processing to analyze the structure of chromatin fibers in human mitotic chromosomes and have proposed the melt model of mitotic chromosome structure (Eltsov et al., 2008). Flexible nucleosomal arrays may fold into $30 \mathrm{~nm}$ chromatin fibers in interphase through intra-array nucleosomal interactions under dilute conditions, and may compact into more condensed structures under high chromatin concentrations in mitosis through inter-array nucleosomal associations. It is not yet clear whether higher-order chromatin structures are formed directly between $30 \mathrm{~nm}$ fibers or via some other intermediate states. More careful experiments are needed to analyze the detailed structure of chromatin fibers under different conditions. Super-resolution optical imaging techniques could potentially also be used to analyze higher-order chromatin structures and their detailed dynamic changes 
during transcription activation and repression in vivo/vitro. Fluorsecence resonance energy transfer (FRET) analysis, a powerful tool for determining intramolecular distances from 2 $\mathrm{nm}$ to $10 \mathrm{~nm}$ in biomolecules has been used to direcly investigate the conformational dynamics of oligonucleosomes. The effect of the linker histone $\mathrm{H} 1$, histone acetylation and salt conditions on the structure and dynamics of trinucleosomes has been studied via bulk solution FRET (Bussiek et al., 2006; Poirier et al., 2009). Lamond and colleagues have adapted multiphoton fluorescence lifetime imaging microscopy (FLIM) with FRET to quantitatively analyze chromatin fiber compaction in living cells (Llères etal., 2009). Furthermore, when extended to the singlemolecule level, FRET can potentially give detailed information on structural distribution, allowing heterogeneous populations and structural substrates to be characterized (Koopmans et al., 2007; Gansen et al., 2009). Although H3K56 acetylation does not affect the compaction of chromatin fibers, quantitative single-molecule via bulk solution FRET analysis on mononucleosomes has revealed its subtle effects by increasing DNA breathing 7-fold at the entry-exit point (Neumann et al., 2009). By selectively labeling donor and acceptor fluorophores on DNA and/or histone proteins, the detailed arrangement of nucleosomes in higher-order chromatin and the dynamics of nucleosomes and chromatins can be explored. We expect that these new techniques will enable us to further understand the structure and dynamics of higherorder chromatin and the underlying mechanisms of gene transcription.

\section{ACKNOWLEDGEMENTS}

This work was supported by the National Natural Science Foundation of China (Grant Nos. 31000566 and 31071147) and the National Basic Research Program (973 Program) of China (Grant No. 2009CB825501).

\section{REFERENCES}

Angelov, D., Verdel, A., An, W., Bondarenko, V., Hans, F., Doyen, C. M., Studitsky, V.M., Hamiche, A., Roeder, R.G., Bouvet, P., et al. (2004). SWI/SNF remodeling and p300-dependent transcription of histone variant $\mathrm{H} 2 \mathrm{ABbd}$ nucleosomal arrays. EMBO J 23, 3815-3824.

Bannister, A.J., Zegerman, P., Partridge, J.F., Miska, E.A., Thomas, J. O., Allshire, R.C., and Kouzarides, T. (2001). Selective recognition of methylated lysine 9 on histone H3 by the HP1 chromo domain. Nature 410, 120-124.

Bussiek, M., Tóth, K., Schwarz, N., and Langowski, J. (2006). Trinucleosome compaction studied by fluorescence energy transfer and scanning force microscopy. Biochemistry 45 , 10838-10846.

Catez, F., Ueda, T., and Bustin, M. (2006). Determinants of histone H1 mobility and chromatin binding in living cells. Nat Struct Mol Biol 13, 305-310.

Dorigo, B., Schalch, T., Kulangara, A., Duda, S., Schroeder, R.R., and Richmond, T.J. (2004). Nucleosome arrays reveal the two-start organization of the chromatin fiber. Science 306, 1571-1573.

Eltsov, M., Maclellan, K.M., Maeshima, K., Frangakis, A.S., and Dubochet, J. (2008). Analysis of cryo-electron microscopy images does not support the existence of 30-nm chromatin fibers in mitotic chromosomes in situ. Proc Natl Acad Sci U S A 105, 19732-19737.

Fan, J.Y., Rangasamy, D., Luger, K., and Tremethick, D.J. (2004). H2A.Z alters the nucleosome surface to promote HP1alphamediated chromatin fiber folding. Mol Cell 16, 655-666.

Francis, N.J., Kingston, R.E., and Woodcock, C.L. (2004). Chromatin compaction by a polycomb group protein complex. Science 306, 1574-1577.

Gansen, A., Valeri, A., Hauger, F., Felekyan, S., Kalinin, S., Tóth, K., Langowski, J., and Seidel, C.A. (2009). Nucleosome disassembly intermediates characterized by single-molecule FRET. Proc Natl Acad Sci U S A 106, 15308-15313.

Hendzel, M.J., Lever, M.A., Crawford, E., and Th'ng, J.P. (2004). The $\mathrm{C}$-terminal domain is the primary determinant of histone $\mathrm{H} 1$ binding to chromatin in vivo. J Biol Chem 279, 20028-20034.

Koopmans, W.J., Brehm, A., Logie, C., Schmidt, T., and van Noort, J. (2007). Single-pair FRET microscopy reveals mononucleosome dynamics. J Fluoresc 17, 785-795.

Li, G., Margueron, R., Hu, G., Stokes, D., Wang, Y.H., and Reinberg, D. (2010). Highly compacted chromatin formed in vitro reflects the dynamics of transcription activation in vivo. Mol Cell 38, 41-53.

Llères, D., James, J., Swift, S., Norman, D.G., and Lamond, A.I. (2009). Quantitative analysis of chromatin compaction in living cells using FLIM-FRET. J Cell Biol 187, 481-496.

Luger, K., Mäder, A.W., Richmond, R.K., Sargent, D.F., and Richmond, T.J. (1997). Crystal structure of the nucleosome core particle at 2.8 A resolution. Nature 389, 251-260.

Neumann, H., Hancock, S.M., Buning, R., Routh, A., Chapman, L., Somers, J., Owen-Hughes, T., van Noort, J., Rhodes, D., and Chin, J.W. (2009). A method for genetically installing site-specific acetylation in recombinant histones defines the effects of $\mathrm{H} 3 \mathrm{~K} 56$ acetylation. Mol Cell 36, 153-163.

Poirier, M.G., Oh, E., Tims, H.S., and Widom, J. (2009). Dynamics and function of compact nucleosome arrays. Nat Struct Mol Biol 16, 938-944.

Robinson, P.J., Fairall, L., Huynh, V.A., and Rhodes, D. (2006). EM measurements define the dimensions of the "30-nm" chromatin fiber: evidence for a compact, interdigitated structure. Proc Natl Acad Sci U S A 103, 6506-6511.

Robinson, P.J., and Rhodes, D. (2006). Structure of the ' $30 \mathrm{~nm}$ ' chromatin fibre: a key role for the linker histone. Curr Opin Struct Biol 16, 336-343.

Routh, A., Sandin, S., and Rhodes, D. (2008). Nucleosome repeat length and linker histone stoichiometry determine chromatin fiber structure. Proc Natl Acad Sci U S A 105, 8872-8877.

Schalch, T., Duda, S., Sargent, D.F., and Richmond, T.J. (2005). X-ray structure of a tetranucleosome and its implications for the chromatin fibre. Nature 436, 138-141.

Shogren-Knaak, M., Ishii, H., Sun, J.M., Pazin, M.J., Davie, J.R., and Peterson, C.L. (2006). Histone H4-K16 acetylation controls chromatin structure and protein interactions. Science 311, 844-847.

Strick, R., Strissel, P.L., Gavrilov, K., and Levi-Setti, R. (2001). Cationchromatin binding as shown by ion microscopy is essential for the structural integrity of chromosomes. J Cell Biol 155, 899-910. 
Thoma, F., Koller, T., and Klug, A. (1979). Involvement of histone H1 in the organization of the nucleosome and of the salt-dependent superstructures of chromatin. J Cell Biol 83, 403-427.

Trojer, P., Li, G., Sims, R.J. 3rd, Vaquero, A., Kalakonda, N., Boccuni, P., Lee, D., Erdjument-Bromage, H., Tempst, P., Nimer, S.D., et al. (2007). L3MBTL1, a histone-methylation-dependent chromatin lock. Cell 129, 915-928.

Watanabe, S., Resch, M., Lilyestrom, W., Clark, N., Hansen, J.C., Peterson, C., and Luger, K. (2010). Structural characterization of H3K56Q nucleosomes and nucleosomal arrays. Biochim Biophys Acta 1799, 480-486.

Widom, J., and Klug, A. (1985). Structure of the 300A chromatin filament: X-ray diffraction from oriented samples. Cell 43, 207-213.

Williams, S.P., Athey, B.D., Muglia, L.J., Schappe, R.S., Gough, A.H., and Langmore, J.P. (1986). Chromatin fibers are left-handed double helices with diameter and mass per unit length that depend on linker length. Biophys J 49, 233-248.

Woodcock, C.L., Frado, L.L., and Rattner, J.B. (1984). The higherorder structure of chromatin: evidence for a helical ribbon arrangement. J Cell Biol 99, 42-52.

Zlatanova, J., Caiafa, P., and Van Holde, K. (2000). Linker histone binding and displacement: versatile mechanism for transcriptional regulation. FASEB J 14, 1697-1704. 